\title{
Encapsulation of temozolomide in a tumor-targeting nanocomplexenhances anti-cancer efficacy and reduces toxicity in a mouse model of glioblastoma
}

Sang-Soo Kim ${ }^{\mathrm{a}}$, Antonina Rait ${ }^{\mathrm{a}}$, Eric Kim ${ }^{\mathrm{b}}$, James DeMarco ${ }^{\mathrm{b}}$, Kathleen F. Pirollo ${ }^{\mathrm{a}}$, Esther H. Chang $^{\mathrm{a}}$

${ }^{a}$ Department of Oncology, Lombardi Comprehensive Cancer Center, Georgetown University, Washington, DC 20057, USA

${ }^{b}$ SynerGene Therapeutics, Inc., Potomac, MD 20854, USA

Corresponding Author: Esther H. Chang, Department of Oncology, Lombardi Comprehensive Cancer Center, Georgetown University Medical Center, 3970 Reservoir Rd. NW, Research Building E420, Washington, DC 20057. Phone: 202-687-8418; Fax: 202-687-8434; E-mail: change@georgetown.edu 


\begin{abstract}
Although temozolomide (TMZ) is the current first-line chemotherapy for glioblastoma multiforme (GBM), most patients either do not respond or ultimately fail TMZ treatment. Both intrinsic tumor resistance and limited access of TMZ to brain tumorsas a result of the blood-brain barrier (BBB) contribute to poor response and ultimately to poor prognosis for GBM patients. We have developed a "dual-targeting" nanomedicine that both actively crosses the BBB and actively targets cancer cells once in the brain parenchyma. This nanomedicine (termed scLTMZ) is sized $\sim 40 \mathrm{~nm}$ and comprised of a cationic liposome (DOTAP:DOPE) encapsulating TMZ. The surface of liposome is decorated with anti-transferrin receptor single-chain antibody fragments to facilitate the crossing of the BBB by the scL-TMZ in addition to targeting GBM in the brain. This novel formulation was found to be markedly more effective than standard TMZ in both TMZ-resistant and TMZ-sensitive GBM. Encapsulation of TMZ also markedly enhanced its efficacy in killing a variety of non-GBM tumor cells. The scL-TMZ nanocomplex was shown to target cancer stem cells, which have been linked to both drug resistance and recurrence in GBM. Most significantly, systemically administered scL-TMZ significantly prolonged survival in mice bearing intracranial GBM tumors. The improved efficacy of scL-TMZ compared to standard TMZ was accompanied by reduced toxicity, so we conclude that the scL-TMZ nanomedicine holds great promise as a more effective therapy for GBM and other tumor types.
\end{abstract}

Keywords:Nanocomplex, Targeted delivery, Temozolomide resistance, Glioblastoma multiforme, Cancer stem cells 
Abbreviations: BBB, blood-brain barrier; CSC, cancer stem cell; GBM, glioblastoma multiforme; H2AX, H2A histone family, member X;scL, tumor-targeted liposome nanocomplex; TMZ, temozolomide; scL-TMZ, scL-encapsulated TMZnanocomplex; TfR, transferrin receptor; TfRscFv, anti-transferrin receptor single-chain antibody fragment. 


\section{Introduction}

Glioblastoma multiforme (GBM) is one of the most lethal forms of cancer. Despite intensive multimodal treatments comprising surgical resection, radiation, and temozolomide (TMZ) chemotherapy, the prognosis of GBM patient is very poor[1].Moreover, approximately $60-75 \%$ of GBM patients do notbenefit from TMZ[2]. Thislack of response has been attributed to a number of factors including, the short half-life of TMZ in circulation, its inefficient crossing of the blood-brain barrier (BBB), poor drug influx andexcessive drug efflux intumor cells, DNA damage repair systems, and deregulation of apoptosis[3,4].Although relatively well tolerated,TMZ possesses dose-limiting side effects [5].

We have developed a tumor-targeting immunoliposomenanocomplex (designated as "scL" referring to it being a liposome with a single chain antibody fragment as a targeting moiety) for systemicdeliveryof its payloadto primary and metastatic tumors with exquisite specificity[6,7].Nanomedicinesbasd on scLcarrying various types of therapeutic payloads (e.g., plasmid DNA, siRNA, and small molecules) have demonstrated anti-tumor effects in a number of preclinical studies [8-12].Furthermore, scLcarrying the wtp53 tumor suppressor gene (in a product termed SGT-53) has successfully completed Phase Ia/Ib clinical trialsdemonstrating its safety and anti-tumoractivity[13].We have recently demonstrated that SGT-53targets cancer stem cells (CSCs)[6].

The tumor-targeting nature of scLnanocomplexis due to an anti-transferrin receptor single-chain antibody fragment (TfRscFv) on its surface, which binds to TfR overexpressed on tumor cells [14].In addition to its overexpression on tumor cells, TfR is instrumental in the process of receptor-mediated transcytosisthat actively moves ligand across the endothelial cells 
that form the BBB. The TfR carries its ligand, diferric transferrin, across the BBB for iron delivery to the brain and this TfR-mediated transcytosis is a well-established pathway. This pathway has been actively investigated to transport therapeutics by linking them either to the ligand or to an antibody recognizing the receptor[15-19].Thus, the scLcan both target the endothelial cells to cross the BBB and target tumor cells once in the brain. We have demonstrated that systemically administered SGT-53 both crosses the BBB and delivers its payload to intracranial tumors[6].Here, we have adapted the scL delivery system to encapsulate TMZ for transcytosis across the BBBand uptake by brain tumor cells.We have compared the efficacy and toxicity of this formulation (termed "scL-TMZ") to conventional TMZ in both cultured cells and mouse models of GBM.

\section{Materialsand Methods}

2.1. Cells

All human cancer cell linesexcept U87-luc2 and U251 wereobtained from American Type Culture Collection.U87-luc2, aluciferase expressing glioblastoma line, was purchased from Caliper Life Sciences.GBM cell line U251 was obtained from Tumor Repository at NCIFrederick. U87R, a TMZ-resistant subclone of U87, was established as previously described[20].

\subsection{Preparation of the scL-TMZ nanocomplex}

Incorporation of $\mathrm{TMZ}$ into cationic liposome was performedusing the ethanol injection method as described previously[21].Fresh TMZ solution was prepared by dissolving TMZ 
(Sigma) in DMSO (Sigma) to a concentration of $20-28 \mathrm{mg} / \mathrm{mL}$. Lipid solution comprising1,2dioleoyl-3-trimethylammonium propane (DOTAP) and dioleolylphosphatidyl ethanolamine (DOPE) (Avanti Polar Lipids) were prepared in ethanol at a molar ratio of 1:1. The same molar ratio of lipid solution and TMZ solution were mixedinto pre-warmed distilled water $\left(65^{\circ} \mathrm{C}\right) \cdot \mathrm{TfR} \underline{\mathrm{scF}} \mathrm{F}$-Lip-TMZ (scL-TMZ) complex was prepared as described previously[22]by mixing the TMZ cationic liposome with TfRscFvsolution wherein the TfRscFv is directly complexed with the cationic liposome.Unencapsulated free TMZ was separated from the TMZ-encapsulated liposome (scL-TMZ) by filtration through Vivaspin 500 (5kDa MWCO, GE Healthcare) and efficiency of encapsulation was calculated. For in vitro experiments, the complex was further diluted with serum-free medium. For animal injections, 50\% dextrose was added to each sample to a final concentration of $5 \%$ dextrose. The size and zeta potential was determined with a ZetasizerNano ZS System (Malvern Instruments).

\subsection{In vitrotransfection and cell viability}

All cell lines were plated at $2.0 \times 10^{3}$ cells/well (96-well plate) and $24 \mathrm{~h}$ later treated with freeTMZ or scL-TMZ. Cells treated with either liposome (Lip) or scLwithout TMZ served as controls. At 72 or $96 \mathrm{~h}$ after the treatment, cell viability was determined by XTTassay and $\mathrm{IC}_{50}$ values, the drug concentration resulting in 50\% cell death, were interpolated from graphs of the drug concentration versus the fraction of surviving cells (SigmaPlot,SystatSoftware).

\subsection{Immunofluorescence imaging}


U87cells were seeded at $5.0 \times 10^{4}$ cells/chamber of Falcon culture slides. After $24 \mathrm{~h}$, cells were treated with either scL-TMZ orunencapsulatedTMZat $100 \mu \mathrm{M}$ for $3 \mathrm{~h}$, after which the media was removed, cells rinsed with PBS, and fresh drug-free media added. At $48 \mathrm{~h}$ posttransfection, cells were fixed,permeabilized, and incubated with rabbit polyclonal anti- $\gamma \mathrm{H} 2 \mathrm{~A}$ histone family, member X (H2AX) (phospho S139) antibody (Abcam) followed by DyLight 488-conjugated donkeyanti-rabbit antibody (BioLegend). Slides were mounted with Vectashield mounting medium with DAPI (Vector Laboratories) and imaged with an Olympus IX71 inverted epi-fluorescent microscopeat 400× magnification.

2.5. Western blot analysis

U87R cells $\left(6.0 \times 10^{5}\right.$ cells $/ 10 \mathrm{~cm}$ dish) were treated with either scL-TMZ or free TMZ at $100 \mu \mathrm{M}$ and harvested 48 and $72 \mathrm{~h}$ post-treatment.Total cellular protein $(40 \mu \mathrm{g})$ was separated on an $8 \%$ SDS-polyacrylamide gel, transferred to nylon membrane, and hybridized with antibodies against human anti- $\gamma \mathrm{H} 2 \mathrm{AX}$ (phospho S139) and cleaved caspase-3 (cCASP3) (Cell Signaling Technology). GAPDHlevels served as protein loadingcontrol. Chemiluminescent detection was performed using ECL (GE Healthcare).

\subsection{Toxicology}

All animal experiments were performed in accordance with approved Georgetown University IACUC protocols. To assess the potential toxicity, 5-6 week old female BALB/c mice (Harlan Sprague-Dawley) were injected via the tail veindaily for 5 consecutive days with scL- 
TMZ or free TMZ, at 15 or $75 \mathrm{mg} / \mathrm{m}^{2}$ TMZ/injection(n=11 per group).On days 7 and 19, blood was collected for hematology and serum chemistry analysis (Antech Diagnostics).

\subsection{Animal models}

For the orthotopic GBM tumor model, 5-6 week old female athymic mice (Harlan Sprague-Dawley) were stereotactically inoculated with U87-luc2 cells as previously described[7]. After 7-10 days, the intracranial tumors were visualized by MRI and tumor volume quantified as previously described[7]. For the subcutaneous GBM tumor model, 5-6 week old female nude mice were inoculated with T98G cells $\left(5.0 \times 10^{6}\right.$ cells/site) suspended in Matrigel (BD Biosciences)[23].Tumor volume $(\mathrm{L} \times \mathrm{W} \times \mathrm{H})$ was determined and treatment began when the tumor averaged $100 \mathrm{~mm}^{3}$.Mice were distributed into treatment groups such that each group began with tumors of similar average size.

\subsection{Bioluminescence imaging}

To measure intracranial U87-luc2 tumor growth, bioluminescence imaging was performed using the Xenogen IVIS ${ }^{\circledR}$ in vivo imaging system (Caliper Life Sciences)[24].

\subsection{Immunohistochemistry}

After completing treatment (day 33) or when the control animals developed excessive tumor burden (day 25), the mice were euthanized. Brains were fixed in $4 \%$ formaldehyde and paraffin embedded. Coronal brain sections were stained with mouse anti-human Ki-67 antibody 
(Dako) plusVectastainABC kit (Vector Laboratories) according to manufacturer's instructions, and imaged using Olympus IX71 microscope (400× magnification). The percentage of Ki-67 positive cells was calculatedin six randomly selected tumor fieldsusing ImageJ software.

\subsection{In vivoefficacy study}

In vivotumor response was assessed by determining the level of apoptosis. At the indicated time, intracranial U87-luc2 tumors and subcutaneous T98G tumors were harvested, weighed, and the tumor cells isolated [6].For the cell cycle assay, cells were fixed in cold ethanol (70\% vol/vol) and stained with propidium iodide (BD Biosciences).For the terminal deoxynucleotidyltransferasedUTP nick end labeling (TUNEL) assay, cells were double-labeled with antibody to human CD133-PE (MiltenyiBiotec), and TUNEL using the in situ cell death detection kit (Roche Applied Science). Apoptosis was further evaluated using cCASP3 and cleaved PARP (cPARP)(Cell Signaling Technology) in combination with CD133-PE and SSEA1-Alexa Fluor 647 (BioLegend). Samples were analyzed by BD FACS Aria flow cytometer (BD Biosciences).

\subsection{Statistical analysis}

Results are presented as the mean \pm SD. The statistical significance was determined using the analysis of variance (ANOVA) (SigmaPlot). $P$ values of $<0.05$ were considered significant. Survival curves were estimated using the Kaplan-Meier method and compared using a log-rank test. 


\section{Results}

\subsection{Characterization of the scL-TMZ nanocomplex}

The mean particle size and zeta potential of scL-TMZare shown in Supplementary Table S1. Adding the tumor-targeting moietyto the liposomes or encapsulation of TMZ slightly increased particle size and decreased zeta potential.However,the scL-TMZ still maintained its nano-scale size $(41.4 \pm 9.2 \mathrm{~nm})$ and positive surface charge $(30.1 \pm 4.6 \mathrm{mV})$. The encapsulation efficiency of TMZ in scL complex was tested 5 times and the average was $45.23 \pm 4.34 \%$.

\section{2. scL-TMZ enhancesGBM killingin vitro}

To assess the anti-tumor activity of scL-TMZ, we evaluated by immunofluorescence the level of $\gamma \mathrm{H} 2 \mathrm{AX}$, which is indicative of double-strand DNA breaks produced by alkylating agent TMZ (Fig. 1A). While treatment with TMZ resulted in DNA damage, significantly more was observed in the nuclei of U87 cells treated with scL-TMZ. To determine if this enhanced DNA damage resulted in increased tumor cell death, XTT cell survival assayswere performed with U87 and U251 cells. In both TMZ-responsive cell lines, scL-TMZ resulted in a 2- to 3-fold increase in cell death as indicated by the changes of $\mathrm{IC}_{50}$ values compared to free TMZ (Fig. 1B and Table 1). In contrast, treatment with Lip alone did not result in any significant cell death. These results demonstrate that there is a substantialincrease in DNA damage and tumor cell death in vitro when TMZ is encapsulated in the scLnanocomplex.

\subsection{In vitrosensitization of TMZ-resistant GBM cells by scL-TMZ}


Since increasing the response of TMZ-resistant GBM tumor would be clinically relevant, U87R, a highly TMZ-resistant subcloneof U87, was tested with scL-TMZ. DNA damage and apoptosis were monitored at 48 and $72 \mathrm{~h}$ after treatment (Fig. 1C). Compared to treatment with free TMZ, the levels of $\gamma \mathrm{H} 2 \mathrm{AX}$ increased by 2- to 3-fold with scL-TMZ. Moreover, increased expression of cCASP3, an indicator of apoptosis, correlated with increased $\gamma \mathrm{H} 2 \mathrm{AX}$, which was enhanced 4.7 -fold $72 \mathrm{~h}$ post-treatment compared to that seen in the cells treated with free TMZ. XTT assay of these TMZ-resistant cells revealed that the cells were considerably more sensitive

to $\mathrm{TMZ}$ when it was formulated asscL-TMZ, reaching $\mathrm{IC}_{50}$ values below $50 \mu \mathrm{M}$ (Fig. 1D) compared to no significant killing of either cell line below a TMZ dose of $500 \mu \mathrm{M}$.This equated to a35-40 fold increase in cell killingbyscL-TMZ. No cell death was observed after treatment withLipat theliposome concentration corresponding to scL-TMZ.

\section{4. scL-TMZ enhances TMZ killing of non-GBM tumor cells}

TMZ is currently being evaluated in clinical trials for treatment of various non-GBM cancers including melanoma, pancreatic, and brain metastases from non-CNS solid tumors (e.g., lung and breast cancers) $[25,26]$. Using the XTT assay, we assessed the potential of scL-TMZ to kill various types of non-GBM cancers in vitro (Table 1 and Supplementary Fig. S1). Human cancer cell lines from melanoma (MDA-MB-435), lung (A549), prostate (DU145), pancreatic (PANC-1), breast (MDA-MB-231) and colon (HT-29) cancers were each treated with either scLTMZ or free TMZ. In all cell lines, scL-TMZ was dramatically more effective in killing the cells than was free TMZ. Treatment with scLvehicle alone which served as control was not effective 
in killing these cells. These results suggest that the potential of scL-TMZ to enhance anti-tumor effect is likely not limited to GBM.

\subsection{Enhanced tumor growth inhibition by scL-TMZ}

Using an intracranial U87-luc2 xenograft model, we examined the anti-tumor effect of systemically administered scL-TMZ in vivo. Animals were treated with either free TMZ or scLTMZ twice/week for 5 weeks as shown in Fig. 2A. Previously in various types of tumors, scLnanocomplex alone(without a payload)did not affect tumor growth. Moreover, we have also observed repeatedly that the untargeted complex ( $\mathrm{scL}$ minus the targeting moiety) does not significantly increase the anti-tumor efficacy of the drugs $[9,27]$. Thus, we did not repeat similar experiments for presentation in this manuscript. Tumor size measured by MRI, luciferase activity from the tumors, and animal survival were monitored up to day 51.After receiving 6 i.v.injections (Day 19), MRI showed a significant inhibition of tumor growth in mice treated with TMZ,as expected for the TMZ-responsive U87-luc2 tumors. However, a significantly greater level of growth inhibition was evident with scL-TMZ (Fig. 2B). Corresponding results were obtained with bioluminescence imaging (Fig. 2C). Based upon quantitation of the tumors' luciferase activities, tumors continued to grow throughout the experiment in all groups except the scL-TMZ treated group (Fig. 2C, lower panel). Although tumor growth was slowed in mice receiving free TMZ, their tumors continued to increase in sizewith rapid tumor growth resuming after treatment ended. In contrast, no significant growth was evident throughout the course of treatment with scL-TMZ, and this inhibition persistedfor $\sim 3$ weeks after the end of treatment. This enhanced inhibitionof tumor growth by scL-TMZ was supported by 
immunohistochemicalstaining of Ki-67, a marker of cell proliferation. Tumors in mice treated with scL-TMZ exhibited a significantly lower percentage of Ki-67-positive cells compared totumors in mice treated with Lip or free TMZ(Fig. 2D).

3.6. Enhanced apoptosis and prolonged survival by scL-TMZ

Using the same experimental design as in Fig. 2A, we evaluated several apoptotic indicators in the tumorsof mice receiving 3 injections (Day 10). Cell cycle analysis showed a significant increase of tumor cells in sub-G1 in thescL-TMZ group (Fig. 3A). Corresponding increasesin indicators of apoptosis (cCASP3, cPARP, and TUNEL) were also observed after treatment with scL-TMZ (Fig. 3B).Importantly, these increased apoptosis indicators were observed in $\mathrm{CD}_{133^{+}} \mathrm{CSC}$ as well as $\mathrm{CD} 133^{-}$bulk tumor cellssuggesting thatscL-TMZ mightreduce chemoresistance and recurrence of GBM mediated by CSCs.

Compared to Lip-treated mice thatall succumbed to disease by day 26 , treatment with free TMZ increased survival by 18 days (Fig. 3C). However, all of these mice also succumbed to disease by day 58 . In contrast, $37.5 \%$ of mice receiving scL-TMZ survived until day 77 (45 days after the end of treatment) when the experiment ended.Mean survival times of mice treated with scL-TMZ was 2.4- and 1.4-fold longerthanthose treated with Lip or free TMZ, respectively. A log-rank statistical analysis showed statistical significance $(P<0.001)$.

3.7. Treatment with scL-TMZ overcomes TMZ-resistance in vivo 
We next examined the ability of systemically administered scL-TMZ to sensitize TMZresistant GBM tumors in micebearing subcutaneous T98G xenograft tumors.Mice were monitored after receiving i.v.injections for 5 days ofeither $200 \mathrm{mg} / \mathrm{m}^{2} /$ day of free $\mathrm{TMZ}$ or 75 $\mathrm{mg} / \mathrm{m}^{2} /$ day of scL-TMZ(Fig. 4A). Although treatment with $200 \mathrm{mg} / \mathrm{m}^{2} /$ day of free TMZ resulted in some tumor growth inhibition during treatment, rapid tumor growth increased post-treatment. In contrast, significant inhibition of tumor growth was evident in mice that received scL-TMZ at $75 \mathrm{mg} / \mathrm{m}^{2} /$ day. On day 18 , tumors in scL-TMZ-treated animals were $55 \%$ smaller than thoseuntreated tumors. Treatment with free TMZ resulted in only $\sim 14 \%$ smaller tumors despite the fact that the amount of drug was $~ 2.7$-fold higher than in the scL-TMZ formulation. Treatment with the $75 \mathrm{mg} / \mathrm{m}^{2} /$ day dose of scL-TMZ also resulted in reduced toxicity as indicated by body weight (Fig. 4B). Thus, use of tumor-targeted scL delivery not only results in improved efficacy at a lower drug dose(even in TMZ-resistant tumors)but also concomitant reduction in toxicity.

The level of apoptosis was determined by TUNEL (Fig. 4C) and cCASP3 staining (Fig. 4D) $48 \mathrm{~h}$ after the end of treatment. Compared to treatment with free $\mathrm{TMZ}$ at $200 \mathrm{mg} / \mathrm{m}^{2} / \mathrm{day}$, a significantlyincreased percent of bulk tumor cells (CD133- or SSEA-1-) as well as CSCs $\left(\mathrm{CD}_{133^{+}}\right.$or SSEA-1 $\left.{ }^{+}\right)$stained positive for TUNEL and cCASP3 in tumors in mice treated withscL-TMZ (at $75 \mathrm{mg} / \mathrm{m}^{2} /$ day).

\subsection{Reduced toxicity associated with scL-TMZ compared tofree TMZ}

Non-tumor-bearing mice were systemically treated with scL-TMZ or free TMZ for 5 days at TMZ doses of 15 and $75 \mathrm{mg} / \mathrm{m}^{2} /$ day. Effects on hematological and biochemical values in 
the blood on day 7 and 19 are shown in Table 2 and Supplementary Table S2, respectively. At a dose of $15 \mathrm{mg} / \mathrm{m}^{2} /$ day, no significant toxic effects were observed with either scL-TMZ or free TMZ on day 7. In animals treated with free $\mathrm{TMZ}$ at $75 \mathrm{mg} / \mathrm{m}^{2} /$ day a $35-45 \%$ decrease in leukocyte, lymphocyte, and thrombocyte counts was observed.In contrast, there was no significant hematological effect in the mice treated with scL-TMZ at $75 \mathrm{mg} / \mathrm{m}^{2} /$ day.Elevation in liver enzymes ALT and AST were also evident after treatment with free TMZ, but not with scLTMZ at $75 \mathrm{mg} / \mathrm{m}^{2} /$ day on day 7. Cardiotoxicity is a known side effect of TMZ [28], and creatine phosphokinase (CPK), an indicator of cardiotoxicity, was elevated $>6$-fold after treatment with free TMZ whereas no increase was observed after treatment with scL-TMZ. Treatment with scL$\mathrm{TMZ}$ at $75 \mathrm{mg} / \mathrm{m}^{2} /$ day resulted in a 2.7 -fold increase in the number of neutrophils on day 7 , but levels of all of the hematological parameters had returned to approximately normal levelsby day 19. In some cases, a minor rebound to slightly higher than normal levels was observed. Collectively, these results demonstrate that encapsulation of TMZ in the scLnanocomplex has the potential to reduce the incidence/severity of leukopenia, lymphopenia, thrombocytopenia in the clinic, well known adverse effects of TMZ treatment[5,29].

\section{Discussion}

Our data indicate that scL-TMZ was capable of killingGBM cells in culture much more effectively than free TMZ. This enhanced killing in vitro reflects the ability of the scLnanocomplex to enter the cancer cells via their cell surface TfR and to deliver a payload (in this case TMZ) to them. Non-GBM cells in culture are also more sensitive to scL-TMZ than to free TMZ. 
For scL-TMZ to be effective in vivo, it must reach the tumor. The difficulty in delivering an effective dose of TMZ to GBM cells in the brain represents a significant obstacle in treatment of the disease. Substantial variability in TMZ response among patientslikely derives in no small measure from the fact that TMZ primarilyenters the brainviadisrupted BBBin the tumor [4], and the degree of disruption varies from patient to patient.The nanomedicine, scL-TMZ, actively crosses the intact BBB by TfR-mediated transcytosis. Once in the brain, scL-TMZ can be actively taken up by GBM cells by the same TfR-mediated mechanism (endocytosis)seen in our in vitro experiments. In a number of our earlier publications, we have repeatedly demonstrated the importance of the targeting moiety for the increased transfection efficiency and tumor targeting ability of the scLnanocomplex when compared to both a control antibody and the unliganded complex in vitro and in vivo[11,30-32].As a result of this dual targeting of both the normal endothelial cells of the BBB and tumor cells in the brain, systemic treatment with scLTMZ significantly improved the anti-tumor activity of TMZ in our animal studies. With both TMZ-sensitive and TMZ-resistant tumors, we observed a substantial inhibition of tumor growth, a substantial increase in cancer cell apoptosis, and a significant prolongation of animal survival. We have shown that, when encapsulated in scL, a TMZ dose that is 2.7 -fold lower than the dose of free TMZ was about 4-fold more effective in controlling tumor growth in the TMZ-resistant tumors suggesting that the improvement of TMZ effectiveness when formulated as an scLnanomedicine is on the order of 10 -fold. This increased effectiveness might enable patients who are less responsive to TMZ to be effectively treated, and may also allow the dose of TMZ to be dropped to reduce side effects.

Other strategies might be combined with scL-TMZ to improve further the efficacy in treating patients having a TMZ-resistant GBM tumor.For example, excessive drug efflux in 
tumor cells can be blocked by inhibiting the efflux pumps such as ATP-binding cassette drug efflux transportersto elevate intracellular TMZ level[33].Another promising strategy is based on inhibition ofthe DNA repair systems such as $\mathrm{O}^{6}$-methylguanine-DNA methyltransferase (MGMT), which repairs the cytotoxic DNA adducts generated by TMZ and increases TMZresistance[4]. In a previous study, we demonstrated that systemic delivery of SGT-53 can abrogate MGMT expression and reverse the TMZ-resistance [6,7]. The combination of SGT-53 and scL-TMZ might further increase anti-tumor efficacy of TMZ in highly TMZ-resistant GBM tumors.

CSCs have been implicated in drug resistance and recurrence of GBM. They have been identified in the infiltrative peritumoral parenchyma of GBM, where the BBB remainsintact (or less altered), resulting in lower accessibility of these regions tofree TMZ[34,35].The restricted bioavailability of free TMZ to CSCs in these areas of tumor infiltration as well as the intrinsic drug-resistance of CSCs may combine to limit the efficacy of current treatment regimens that utilize free TMZ.In this study, we demonstrated substantial induction of apoptosis by scL-TMZ in vivo in $\mathrm{CD}_{133^{+}}$or SSEA $-1^{+} \mathrm{CSC}$ in the tumors. This result suggests that the ability of scLTMZ to reach and affect CSCs could lead to a decrease in tumor recurrence and the development of resistance in initially TMZ-sensitive GBM tumors and to help overcome chemoresistance in TMZ-resistant GBM tumors.

Another significant findings presented here are the results of our toxicology studies. Therapeutic dosages of TMZ are currently limited by the potential for developing myelosuppressive disorders, primarily neutropenia and thrombocytopenia[5,29]. Incidences of opportunistic infections, e.g. pneumocystis pneumonia, have also been associated with long-term usage of TMZ[36]. Ourtoxicologystudy comparingscL-TMZ and free TMZ demonstrated that in 
its nanomedicine formulation TMZ-associated toxicities were significantly reduced. Generally, GBM patients receive multiple cycles of TMZ treatment. However, treatment can be interrupted or discontinued based upon the severity of the side effects. A clear advantage of scL-TMZ is the potential to reduce the incidence of these adverse reactions, not only increasing the patients' quality-of-life on treatment, but also extending the duration of treatment and improving the probability of prolonged survival.

In summary, our data indicate that systemic delivery of TMZ viaa nanomedicine formulation ( $\mathrm{scL}$ ) provides a means both to enhance the therapeutic efficacy of $\mathrm{TMZ}$ and to reduce TMZ-related toxicities. Theabilitiesof this dually targeted nanomedicineto cross the BBB and to target tumorswith a high degree of specifically may result in an increased response rate in GBM patientsand a significant reduction in toxicity. Thus, scL-TMZ could lead to a significant improvement in the prognosis of GBM patients, and this tumor-targeting nanomedicinethat also has the potential for use against a broad range of non-GBM cancers.

\section{Conflict of interest}

Drs. Chang, Pirollo, Rait, and Kim are four of the inventors of the described technology, for which several patents owned by Georgetown University have been issued. The patents have been licensed to SynerGene Therapeutics, Inc. for commercial development. Dr. Chang owns equity interests in SynerGene Therapeutics, Inc. and serves as a non-paid scientific consultant to SynerGene Therapeutics, Inc. 


\section{Acknowledgements}

The authors thank Joe B. Harford for his editorial assistance in preparing this manuscript. We thank Karen Creswell for her technical assistance with flow cytometry. We also thank Olga C. Rodriguez and YiChien Lee for their assistance with MRI imaging and analysis. This study was supported in part by the NCI (5R01CA132012-02 to EHC), NCI SBIR contract (HHSN261201400038C to SynerGene Therapeutics), the National Foundation for Cancer Research (HU0001 to EHC), and a research grant from SynerGene Therapeutics Inc. (to KFP). This study was conducted in part using the Flow Cytometry \& Cell Sorting Shared Resource, Preclinical Imaging Research Laboratory, Microscopy \& Imaging Shared Resource, Tissue Culture \& Biorepository for Cell Lines and Biofluids Shared Resource, and Animal Core Facilities supported by NCI Cancer Center Support grant (2P30CA51008) and U.S. Public Health Service grant (1S10RR15768-01). This investigation was performed in part in a facility constructed with support from Research Facilities Improvement grant from the National Center for Research Resources, NIH (C06RR14567).

\section{Conflict of interest statement}

None 


\section{References}

[1] D.T. Nagasawa, et al., Temozolomide and other potential agents for the treatment of glioblastoma multiforme, Neurosurg Clin N Am 23 (2012) 307-322, ix.

[2] M.C. Chamberlain, Temozolomide: therapeutic limitations in the treatment of adult highgrade gliomas, Expert Rev Neurother 10 (2010) 1537-1544.

[3] Y.P. Ramirez, J.L. Weatherbee, R.T. Wheelhouse, A.H. Ross, Glioblastoma multiforme therapy and mechanisms of resistance, Pharmaceuticals (Basel) 6 (2013) 1475-1506.

[4] D. Beier, J.B. Schulz, C.P. Beier, Chemoresistance of glioblastoma cancer stem cells--much more complex than expected, Mol Cancer 10 (2011) 128.

[5] A. Berrocal, et al., Extended-schedule dose-dense temozolomide in refractory gliomas, J Neurooncol 96 (2010) 417-422.

[6] S.S. Kim, et al., A nanoparticle carrying the p53 gene targets tumors including cancer stem cells, sensitizes glioblastoma to chemotherapy and improves survival, ACS Nano 8 (2014) 54945514.

[7] S.S. Kim, A. Rait, E. Kim, K.F. Pirollo, E.H. Chang, A tumor-targeting p53 nanodelivery system limits chemoresistance to temozolomide prolonging survival in a mouse model of glioblastoma multiforme, Nanomedicine 11 (2015) 301-311.

[8] K.F. Pirollo, et al., p53 mediated sensitization of squamous cell carcinoma of the head and neck to radiotherapy, Oncogene 14 (1997) 1735-1746.

[9] K.F. Pirollo, et al., Tumor-targeting nanocomplex delivery of novel tumor suppressor RB94 chemosensitizes bladder carcinoma cells in vitro and in vivo, Clin Cancer Res 14 (2008) 21902198. 
[10] A.S. Rait, K.F. Pirollo, L. Xiang, D. Ulick, E.H. Chang, Tumor-targeting, systemically delivered antisense HER-2 chemosensitizes human breast cancer xenografts irrespective of HER2 levels, Mol Med 8 (2002) 475-486.

[11] L. Xu, K.F. Pirollo, E.H. Chang, Tumor-targeted p53-gene therapy enhances the efficacy of conventional chemo/radiotherapy, J Control Release 74 (2001) 115-128.

[12] L. Xu, K.F. Pirollo, W.H. Tang, A. Rait, E.H. Chang, Transferrin-liposome-mediated systemic p53 gene therapy in combination with radiation results in regression of human head and neck cancer xenografts, Hum Gene Ther 10 (1999) 2941-2952.

[13] N. Senzer, et al., Phase I Study of a Systemically Delivered p53 Nanoparticle in Advanced Solid Tumors, Mol Ther 21 (2013) 1096-1103.

[14] T.R. Daniels, T. Delgado, G. Helguera, M.L. Penichet, The transferrin receptor part II: targeted delivery of therapeutic agents into cancer cells, Clin Immunol 121 (2006) 159-176.

[15] A.R. Jones, E.V. Shusta, Blood-brain barrier transport of therapeutics via receptormediation, Pharm Res 24 (2007) 1759-1771.

[16] K. Ulbrich, T. Hekmatara, E. Herbert, J. Kreuter, Transferrin- and transferrin-receptorantibody-modified nanoparticles enable drug delivery across the blood-brain barrier (BBB), Eur J Pharm Biopharm 71 (2009) 251-256.

[17] W.M. Pardridge, R.J. Boado, Reengineering biopharmaceuticals for targeted delivery across the blood-brain barrier, Methods Enzymol 503 (2012) 269-292.

[18] C. Dufes, M. Al Robaian, S. Somani, Transferrin and the transferrin receptor for the targeted delivery of therapeutic agents to the brain and cancer cells, Ther Deliv 4 (2013) 629640. 
[19] J. Kreuter, Mechanism of polymeric nanoparticle-based drug transport across the bloodbrain barrier (BBB), J Microencapsul 30 (2013) 49-54.

[20] S. Kohsaka, et al., STAT3 inhibition overcomes temozolomide resistance in glioblastoma by downregulating MGMT expression, Mol Cancer Ther 11 (2012) 1289-1299.

[21] L. Xu, et al., Systemic tumor-targeted gene delivery by anti-transferrin receptor scFvimmunoliposomes, Mol Cancer Ther 1 (2002) 337-346.

[22] W. Yu, et al., Enhanced transfection efficiency of a systemically delivered tumor-targeting immunolipoplex by inclusion of a $\mathrm{pH}$-sensitive histidylated oligolysine peptide, Nucleic Acids Res 32 (2004) e48.

[23] B.A. Teicher, et al., Antiangiogenic and antitumor effects of a protein kinase Cbeta inhibitor in human T98G glioblastoma multiforme xenografts, Clin Cancer Res 7 (2001) 634-640.

[24] W. Yang, et al., ERK1/2-dependent phosphorylation and nuclear translocation of PKM2 promotes the Warburg effect, Nat Cell Biol 14 (2012) 1295-1304.

[25] W. Zhu, et al., Temozolomide for treatment of brain metastases: A review of 21 clinical trials, World J Clin Oncol 5 (2014) 19-27.

[26] Z. Tatar, et al., Temozolomide and unusual indications: review of literature, Cancer Treat Rev 39 (2013) 125-135.

[27] L. Xu, et al., Systemic p53 gene therapy of cancer with immunolipoplexes targeted by antitransferrin receptor scFv, Mol Med 7 (2001) 723-734.

[28] G. Huang, N. Zhang, X. Bi, M. Dou, Solid lipid nanoparticles of temozolomide: potential reduction of cardial and nephric toxicity, Int J Pharm 355 (2008) 314-320.

[29] S. Sengupta, J. Marrinan, C. Frishman, P. Sampath, Impact of temozolomide on immune response during malignant glioma chemotherapy, Clin Dev Immunol 2012 (2012) 831090. 
[30] W. Yu, et al., A sterically stabilized immunolipoplex for systemic administration of a therapeutic gene, Gene Ther 11 (2004) 1434-1440.

[31] L. Xu, et al., Self-assembly of a virus-mimicking nanostructure system for efficient tumortargeted gene delivery, Hum Gene Ther 13 (2002) 469-481.

[32] C. Yang, et al., Nanoimmunoliposome delivery of superparamagnetic iron oxide markedly enhances targeting and uptake in human cancer cells in vitro and in vivo, Nanomedicine 4 (2008) 318-329.

[33] C.W. Chou, et al., Tumor cycling hypoxia induces chemoresistance in glioblastoma multiforme by upregulating the expression and function of ABCB1, Neuro Oncol 14 (2012) 1227-1238.

[34] L. Juillerat-Jeanneret, The targeted delivery of cancer drugs across the blood-brain barrier: chemical modifications of drugs or drug-nanoparticles?, Drug Discov Today 13 (2008) 10991106.

[35] S. Noell, D. Mayer, W.S. Strauss, M.S. Tatagiba, R. Ritz, Selective enrichment of hypericin in malignant glioma: pioneering in vivo results, Int J Oncol 38 (2011) 1343-1348.

[36] L. Tentori, G. Graziani, Recent approaches to improve the antitumor efficacy of temozolomide, Curr Med Chem 16 (2009) 245-257. 


\section{Figure Legends}

Fig. 1. Enhanced cell death by the scL-TMZin vitro. (A)DNA damage was monitored by detecting $\gamma \mathrm{H} 2 \mathrm{AX}$ formation, which is indicative of double-strand DNA breaks produced by alkylating agent TMZ, $48 \mathrm{~h}$ post-treatment. Scale bars indicate $20 \mu \mathrm{m}$.(B) XTT assay in TMZsensitive cells $96 \mathrm{~h}$ post-treatment. (C)Western blot analysis detecting $\gamma \mathrm{H} 2 \mathrm{AX}$ and cCASP3 in TMZ-resistant U87R cells after treatment with either scL-TMZ or TMZ (at $100 \mu \mathrm{M}$ for $3 \mathrm{~h}$ ). (D)XTT assay in TMZ-resistant cells $72 \mathrm{~h}$ post-treatment. Cells treated with Lip alone served as a control.

Fig.2. Enhanced tumor growth inhibition by scL-TMZ in U87-luc2 intracranial xenografts. (A)Treatment schedule. (B) Representative MR images (upper panel) and quantification (lower panel) of tumor volume. Red lines indicate the outline of brain tumors. $* P<0.05$ in lower panel. (C) Representative bioluminescence images shown in a color map: Red $=$ stronger signal, Violet $=$ weaker signal. Three mice from each treatment group are shown fromthe indicated time points. Bioluminescence intensity plotted as a function of time (lower panel). Bar indicates duration of treatment. (D) Representative immunohistochemical staining (right panel) and quantification (left panel) of Ki-67 of the brain tumors on day $33 .^{\dagger}$ Day 25 for Lip group. $* P<0.05, * * P<0.001$. Scale bars indicate $50 \mu \mathrm{m}$.

Fig.3. Prolonged survival and enhanced apoptosis after treatment with scL-TMZ in U87-luc2 intracranial xenografts. Mice were treated as shown in Fig.2A. (A)Quantification of the apoptotic 
response represented by the percent of cells in the sub-G1 population on day $10 . * P<0.05$, $* * P<0.001$. (B)Quantification of apoptosis in $\mathrm{CD} 133^{+} \mathrm{CSCs}$ and $\mathrm{CD} 133^{-}$bulk tumor cells by cCASP3, cPARP, and TUNEL staining on day 10. (C)Kaplan-Meier survival curves. Bar indicates duration of treatment. $\mathrm{n}=7 \sim 8$.

Fig.4.In vivo sensitization of TMZ-resistant T98G tumors by scL-TMZ. Mice bearing subcutaneous T98G tumor xenografts were randomized to therapy with scL-TMZ or free TMZ. Tumor growth (A) and body weight (B) over time. Arrows indicate treatment days. $n=10$ group, $* P<0.05, * * P<0.001$. (C) Quantification of apoptosis in CD133 ${ }^{+}$CSCs and CD133- bulk tumor cells by TUNEL assay performed on day 7. Values indicate the percent of TUNEL positive cells. (D) Quantification of the apoptotic response in SSEA-1 ${ }^{+}$CSCs and SSEA-1 ${ }^{-}$bulk tumor cells represented by the percent of tumor cells positive for cCASP3 staining on day 7 . Values indicate the percentages of cCASP 3 positive cells. 
Figure 1

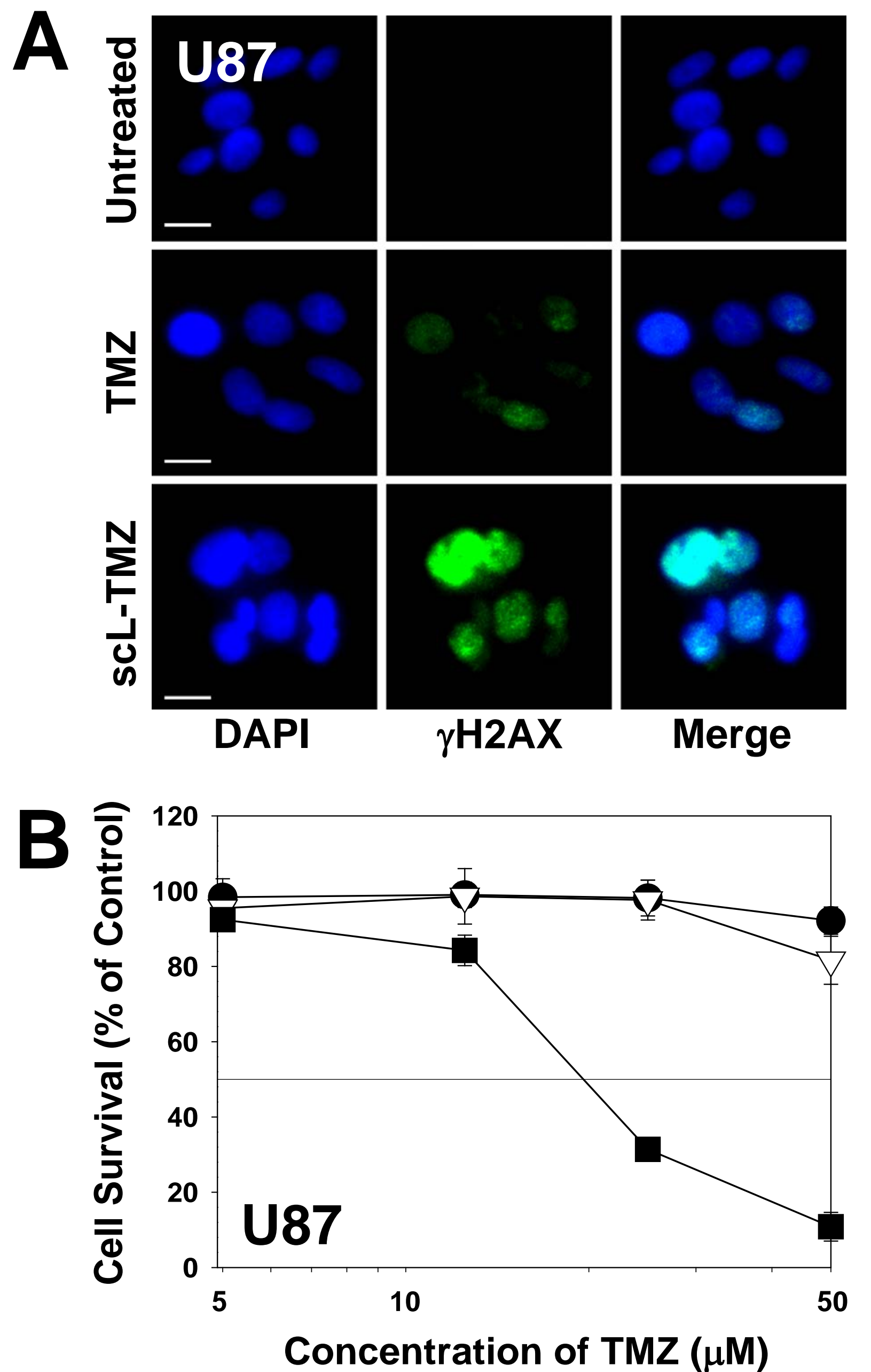

$\mathrm{IC}_{50}$

- Lip $>50.0 \mu \mathrm{M}$

$\rightarrow$ TMZ $>50.0 \mu \mathrm{M}$

- ScL-TMZ $19.6 \mu \mathrm{M}$

D

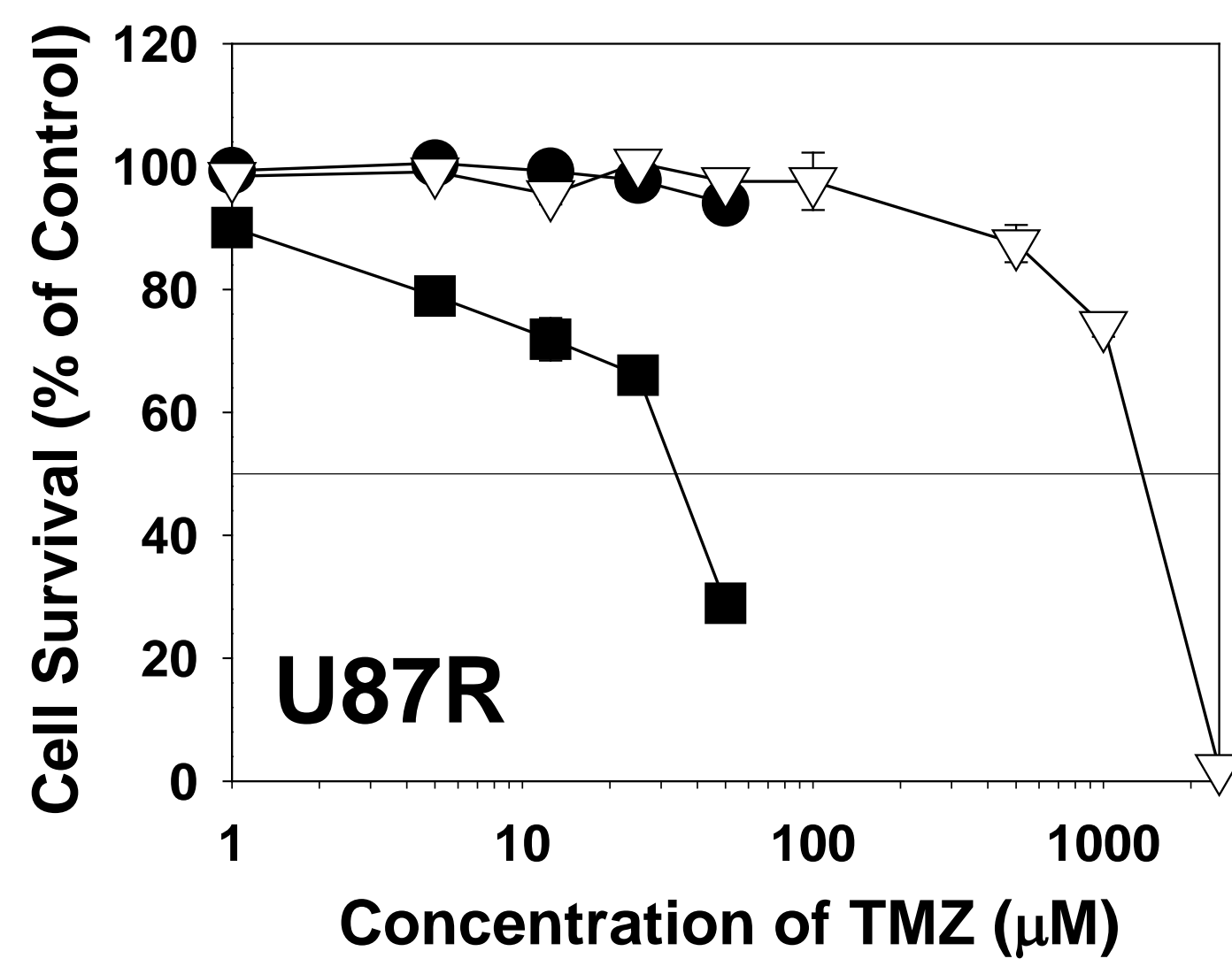

$\mathrm{IC}_{50}$

- L Lip $\quad>50.0 \mu \mathrm{M}$

$\neg$ TMZ $\quad 1356.9 \mu \mathrm{M}$

- scL-TMZ $33.6 \mu \mathrm{M}$
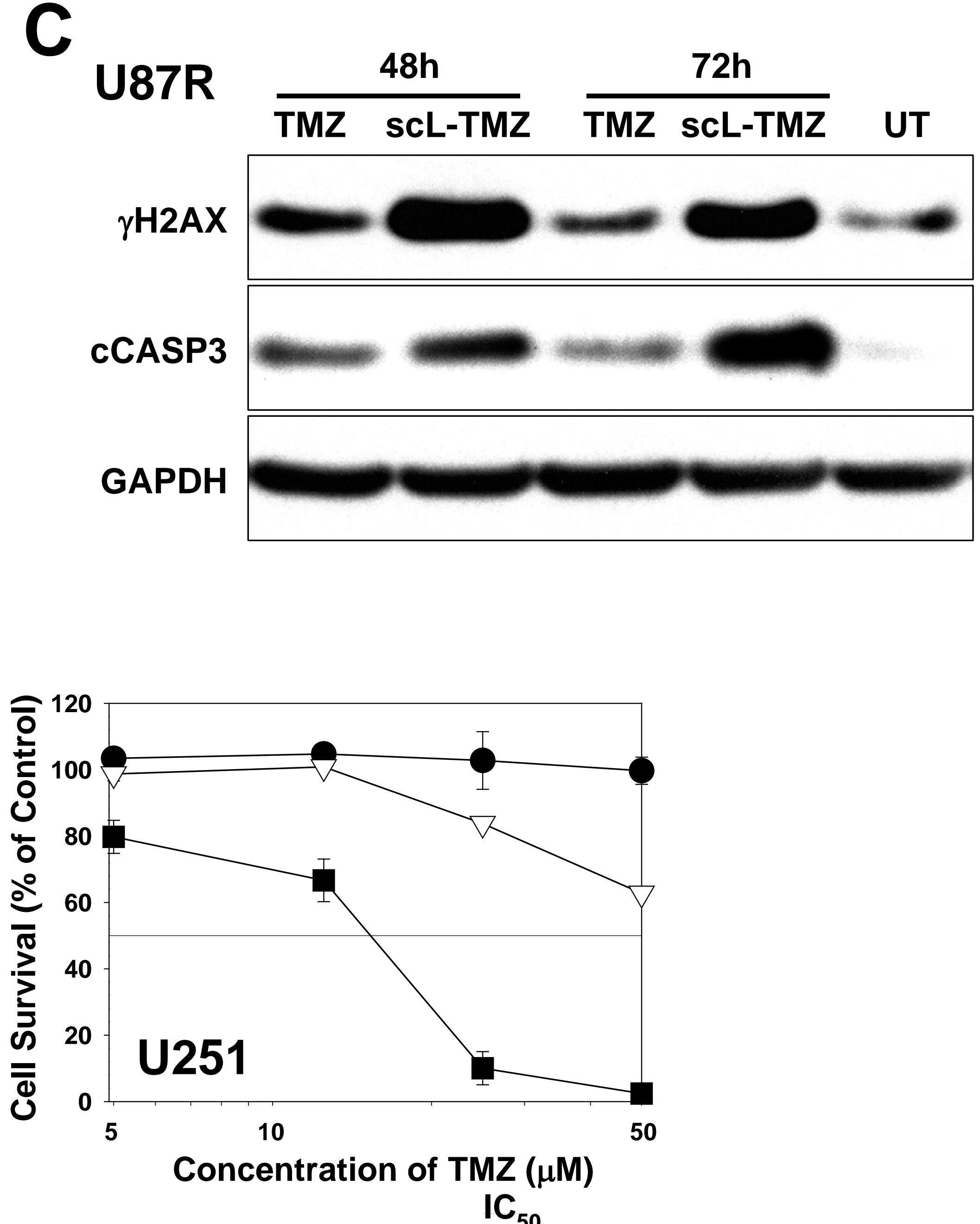

- Lip $\quad>50.0 \mu \mathrm{M}$

$-\nabla \mathrm{TMZ}>50.0 \mu \mathrm{M}$

- scL-TMZ $15.3 \mu \mathrm{M}$

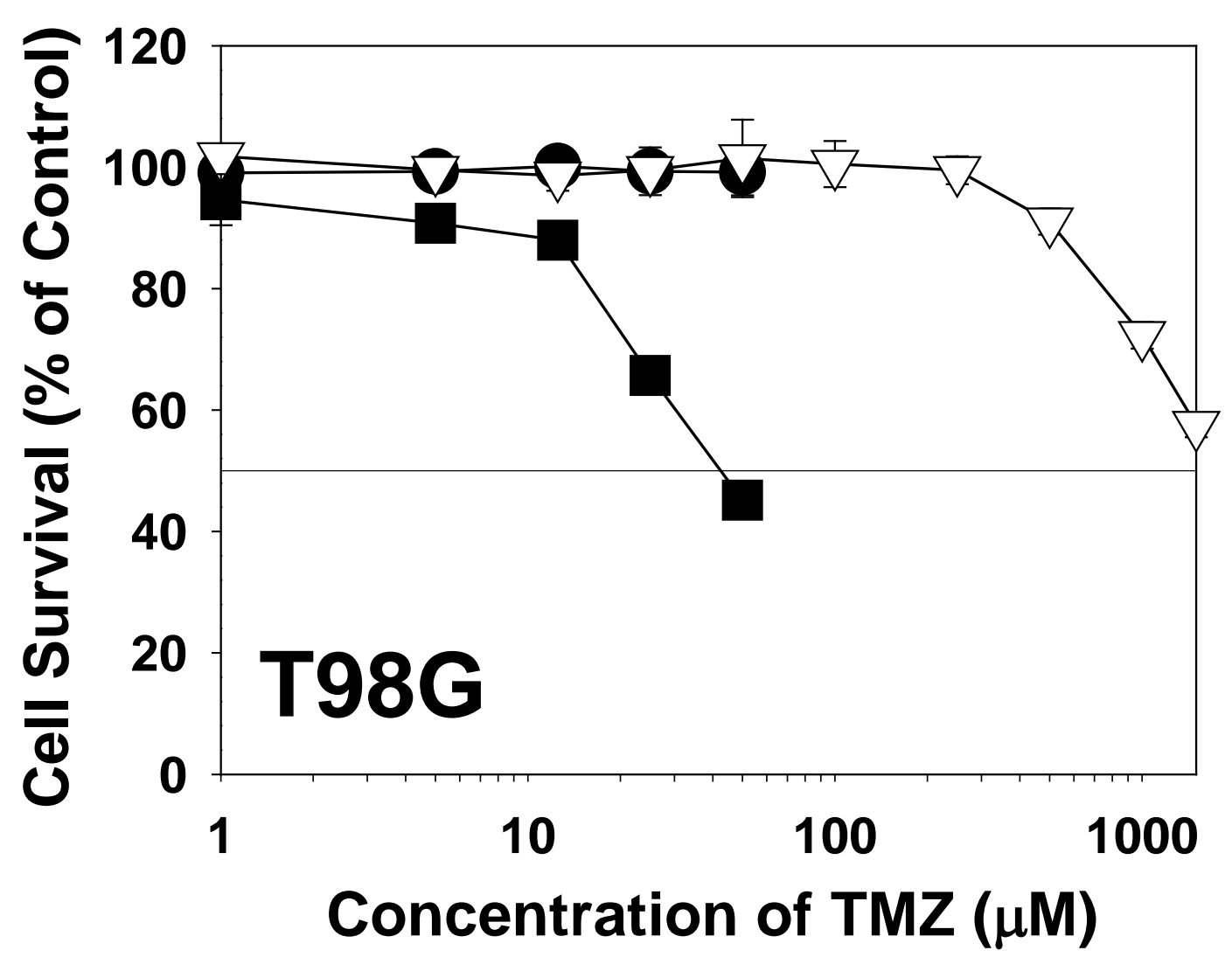

$\mathrm{IC}_{50}$

- Lip $\quad>50.0 \mu \mathrm{M}$

$\rightarrow-\mathrm{TMZ} \quad>1500.0 \mu \mathrm{M}$

- ScL-TMZ $\quad 42.4 \mu \mathrm{M}$ 
Figure 2

\begin{tabular}{|c|c|c|c|c|c|c|c|c|c|c|c|c|c|c|}
\hline$\Lambda$ & & $\begin{array}{c}\mathrm{MRI} \\
\downarrow\end{array}$ & & & & & & & $\begin{array}{c}\text { MRI } \\
\downarrow\end{array}$ & & & & & \\
\hline \multirow[t]{2}{*}{-10} & -7 & 0 & 1 & 4 & 8 & 11 & 15 & 18 & 19 & 22 & 25 & 29 & 32 & Days \\
\hline & & & $\uparrow$ & $\uparrow$ & $\uparrow$ & $\uparrow$ & $\uparrow$ & $\uparrow$ & & $\uparrow$ & $\uparrow$ & $\uparrow$ & $\uparrow$ & \\
\hline \multicolumn{3}{|c|}{$\begin{array}{l}\text { U87-luc2 } \\
\text { inoculation (i.c.) }\end{array}$} & & & & & & & & & . & & & \\
\hline
\end{tabular}
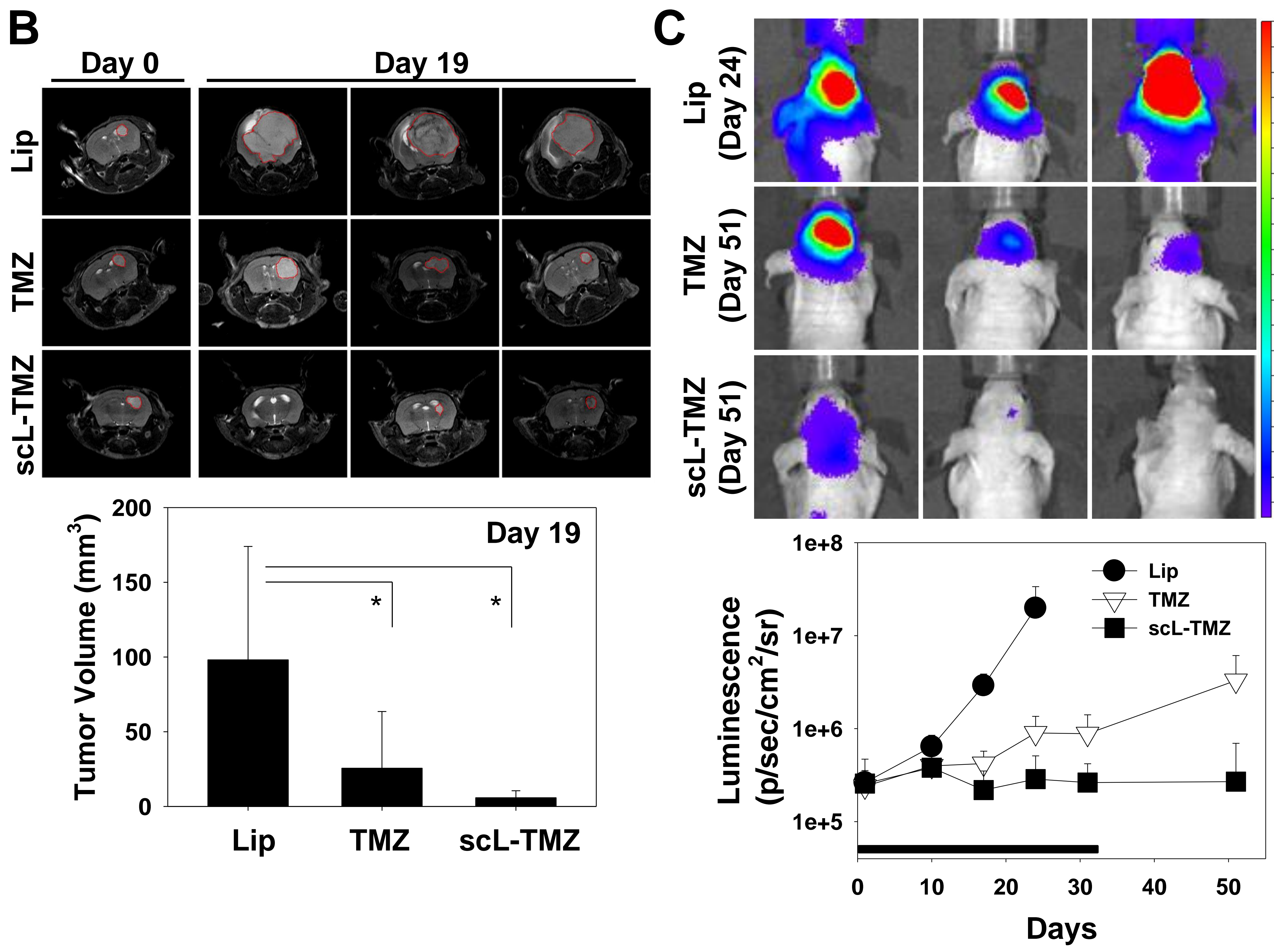

D

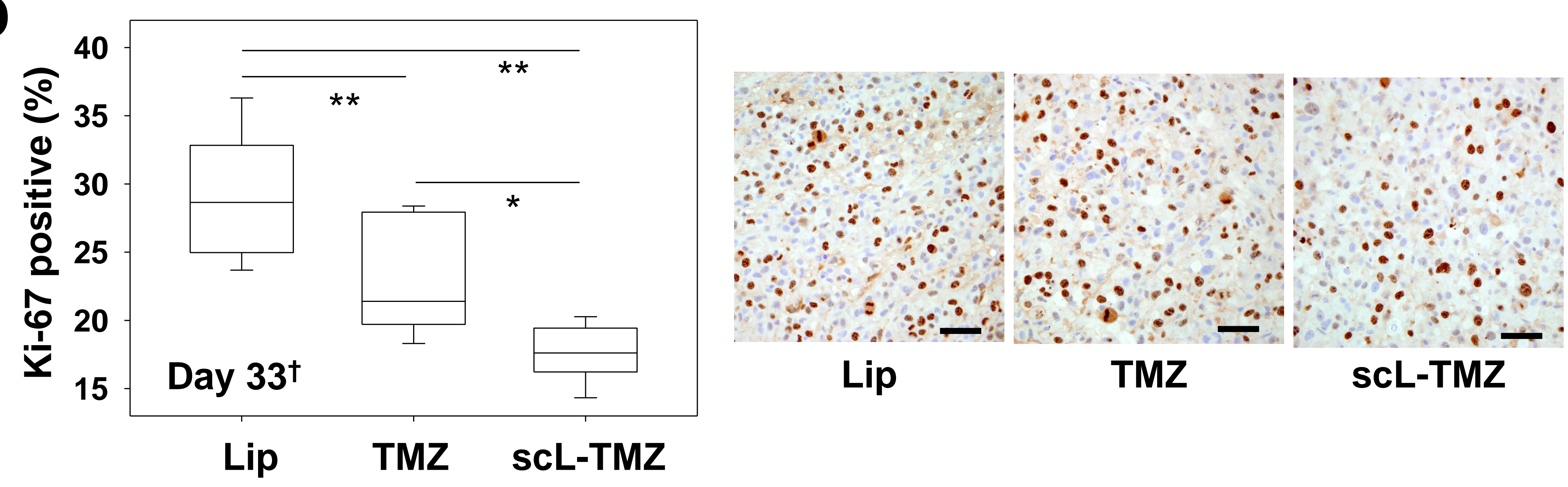


Figure 3

A

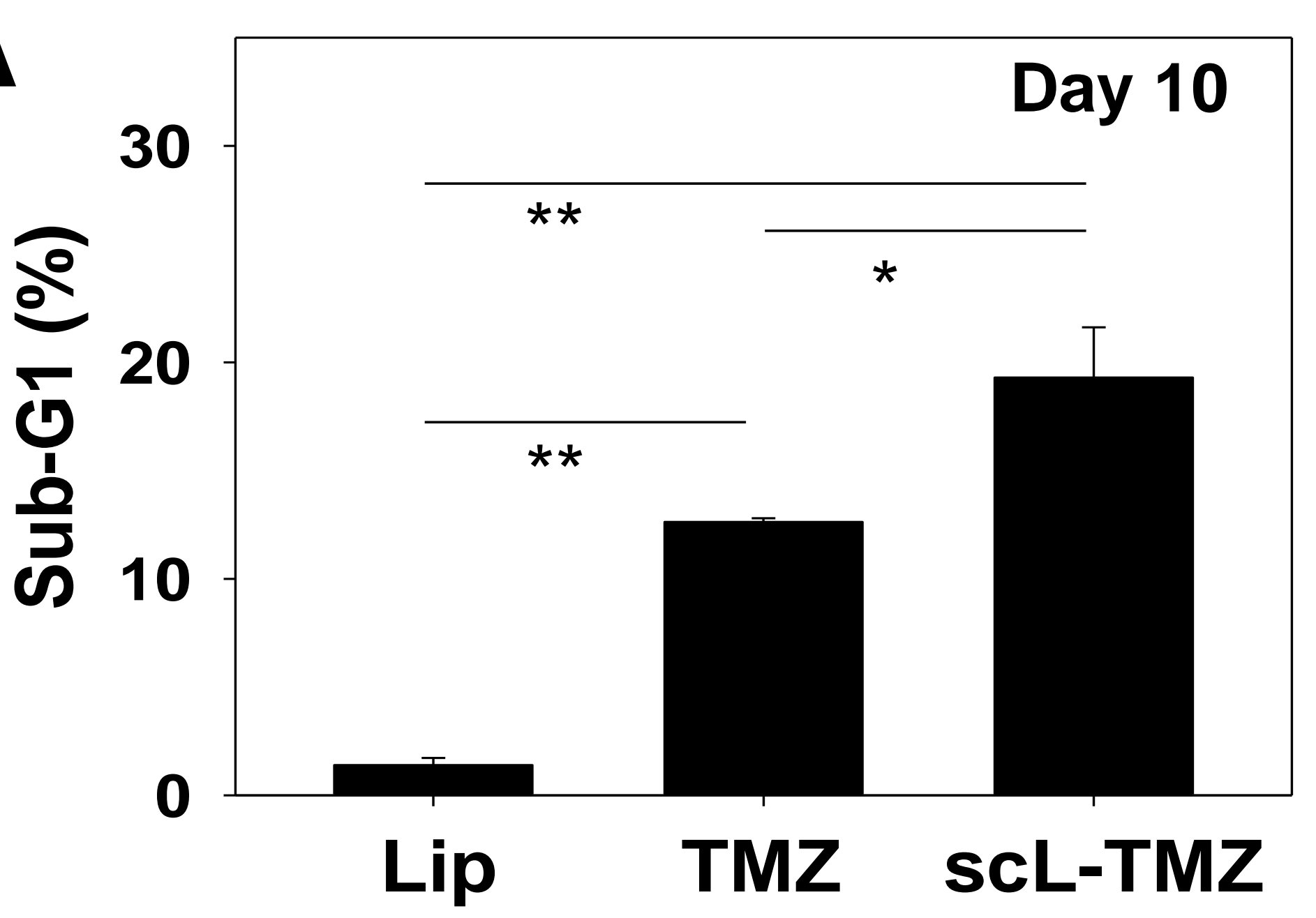

C

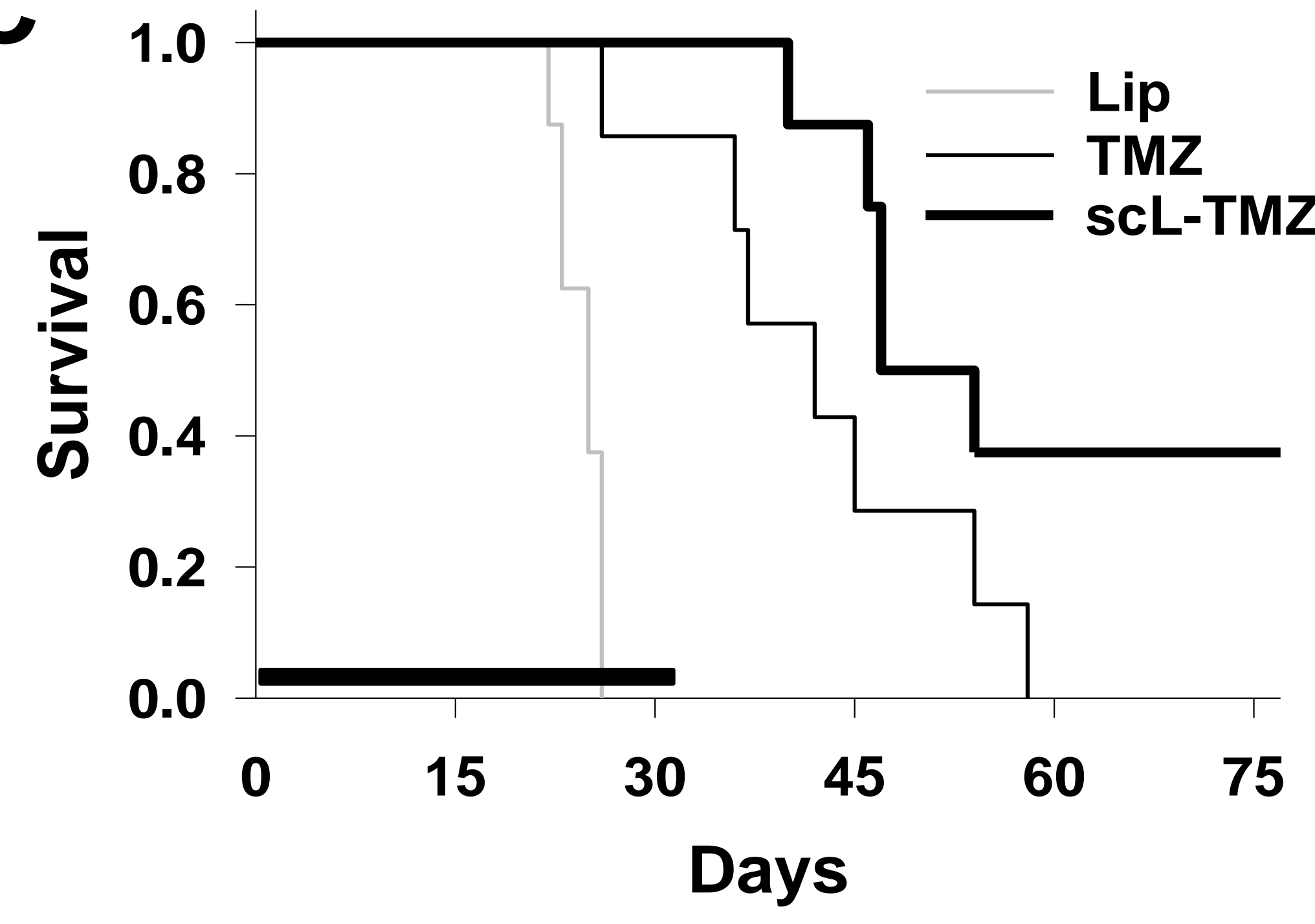

B
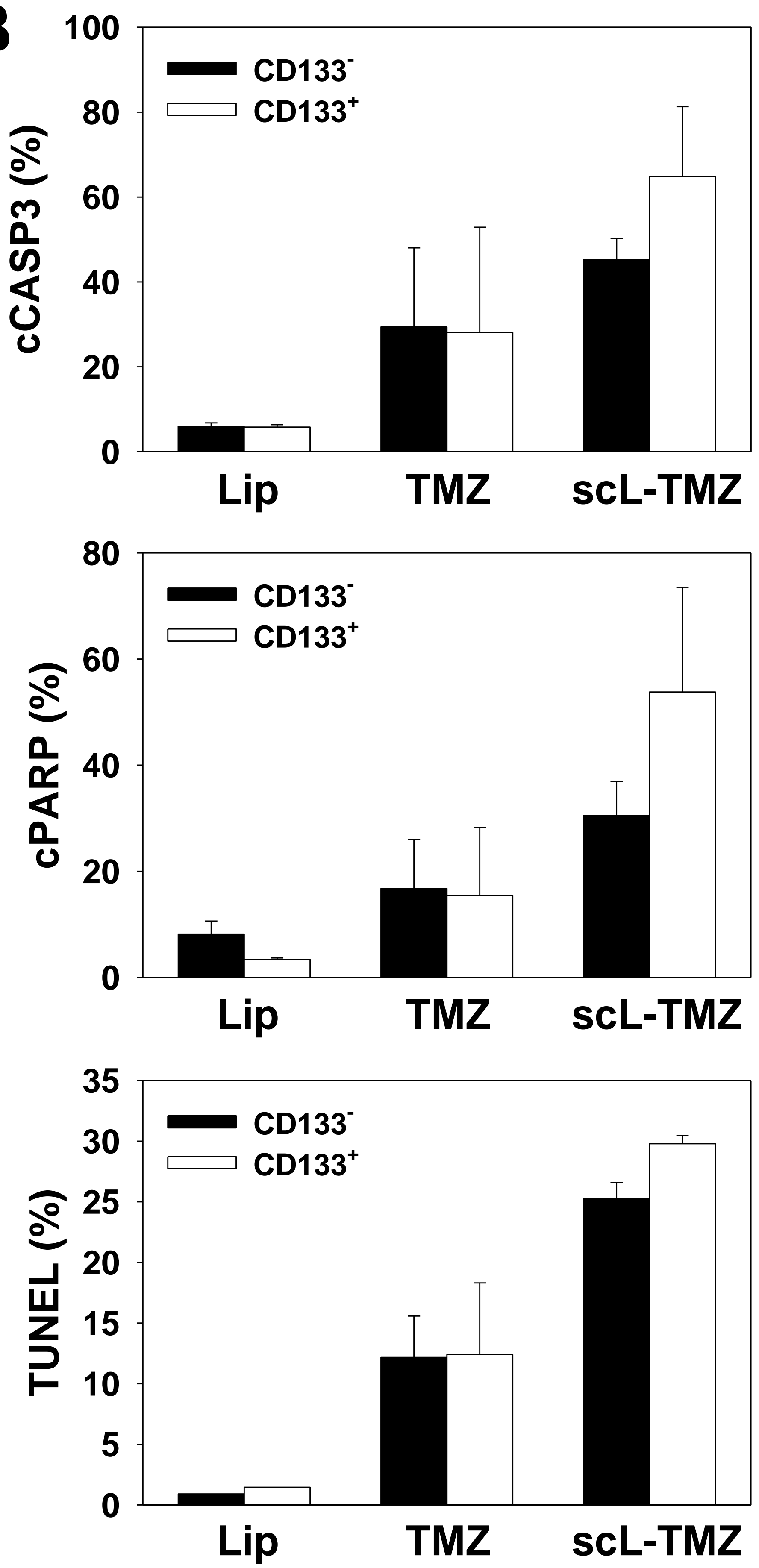
Figure 4

A

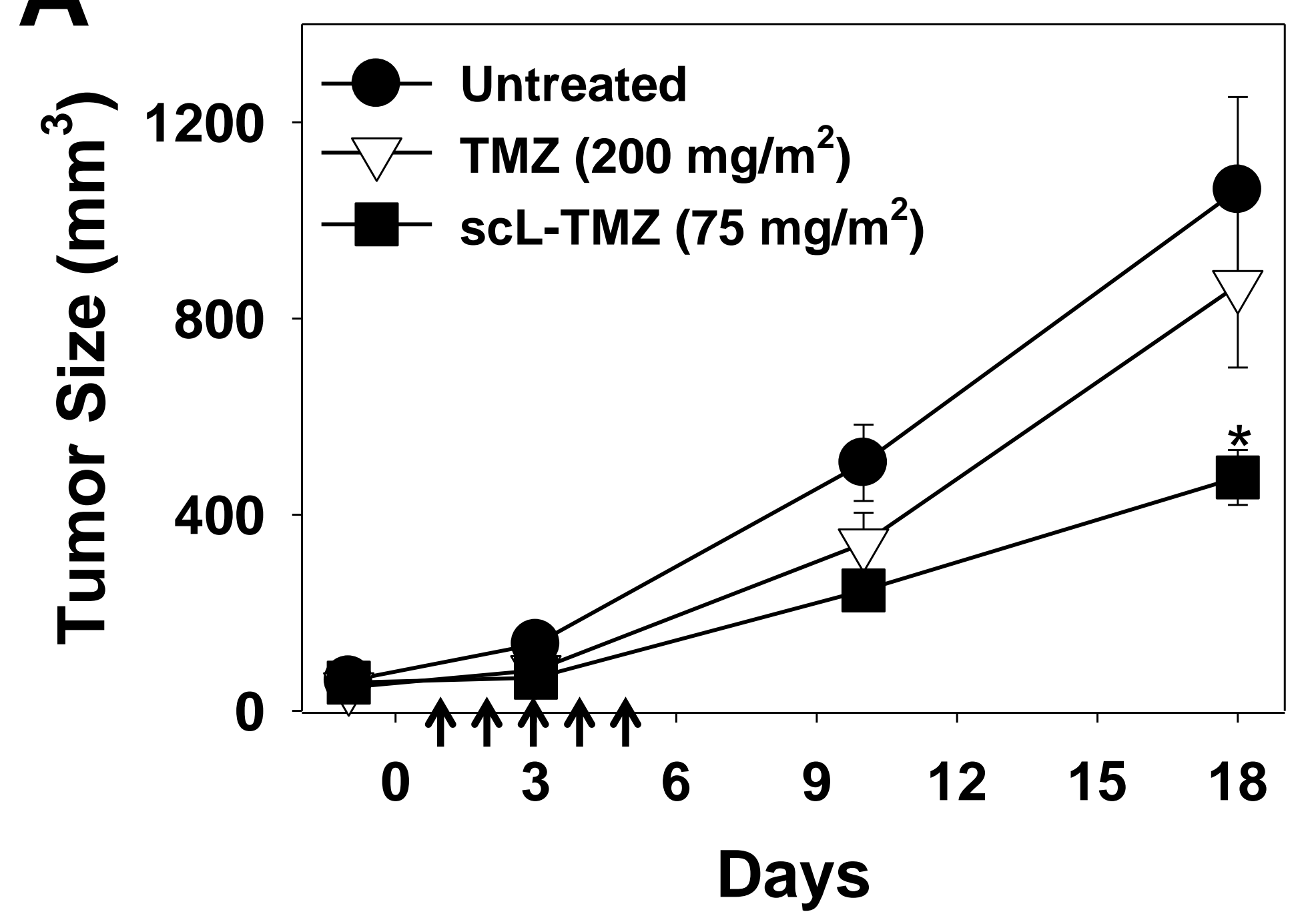

B

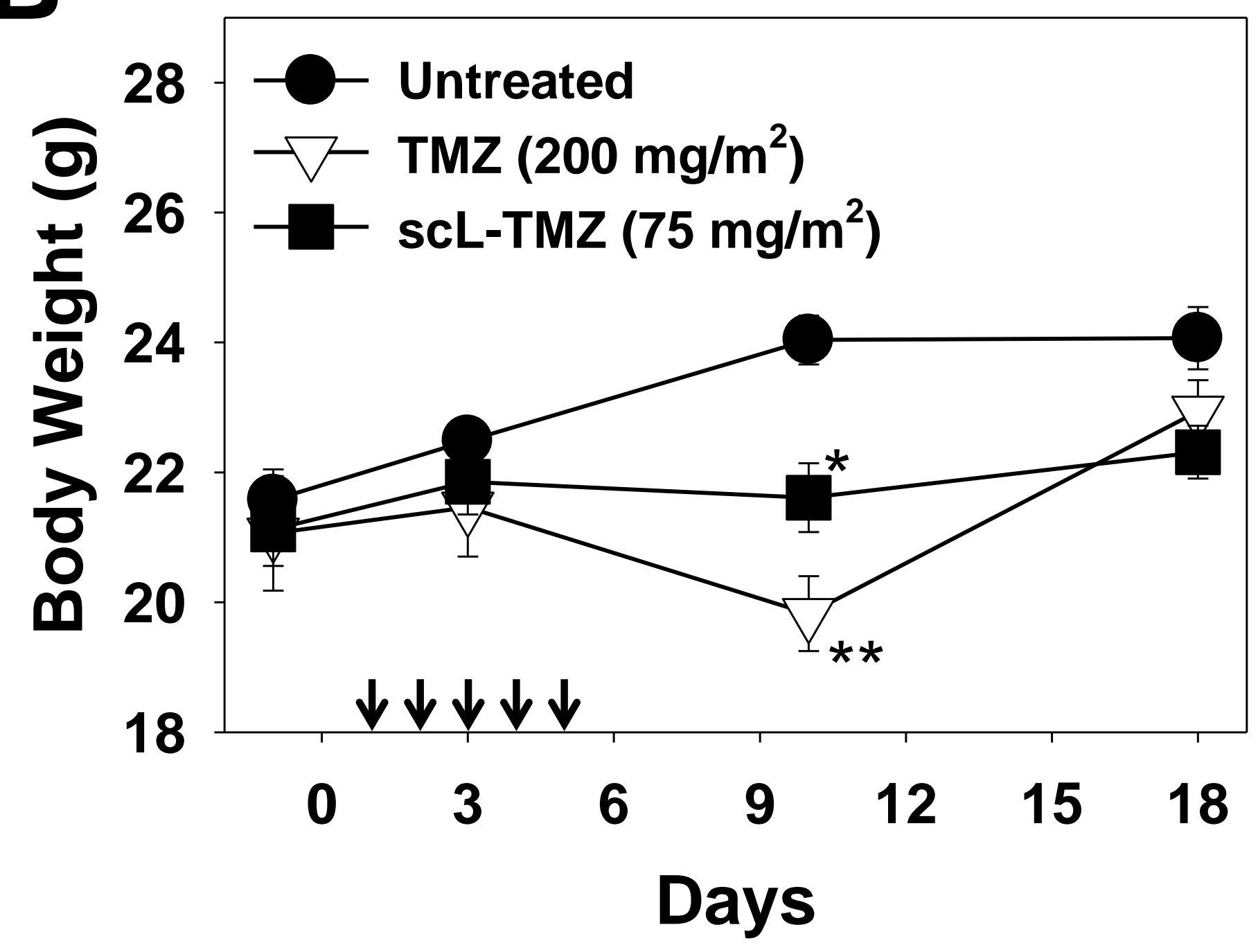

C

CD133-

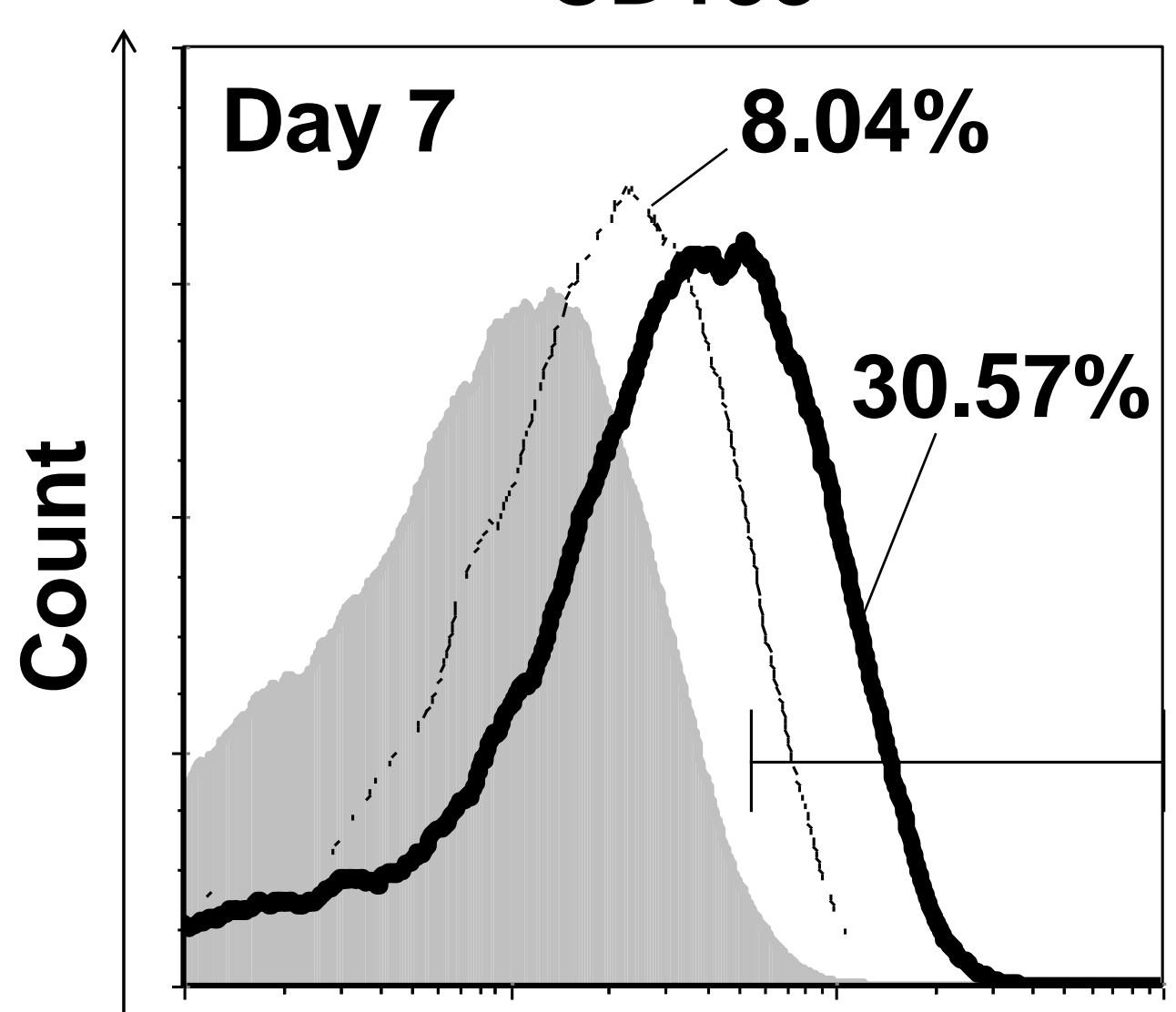

CD133+

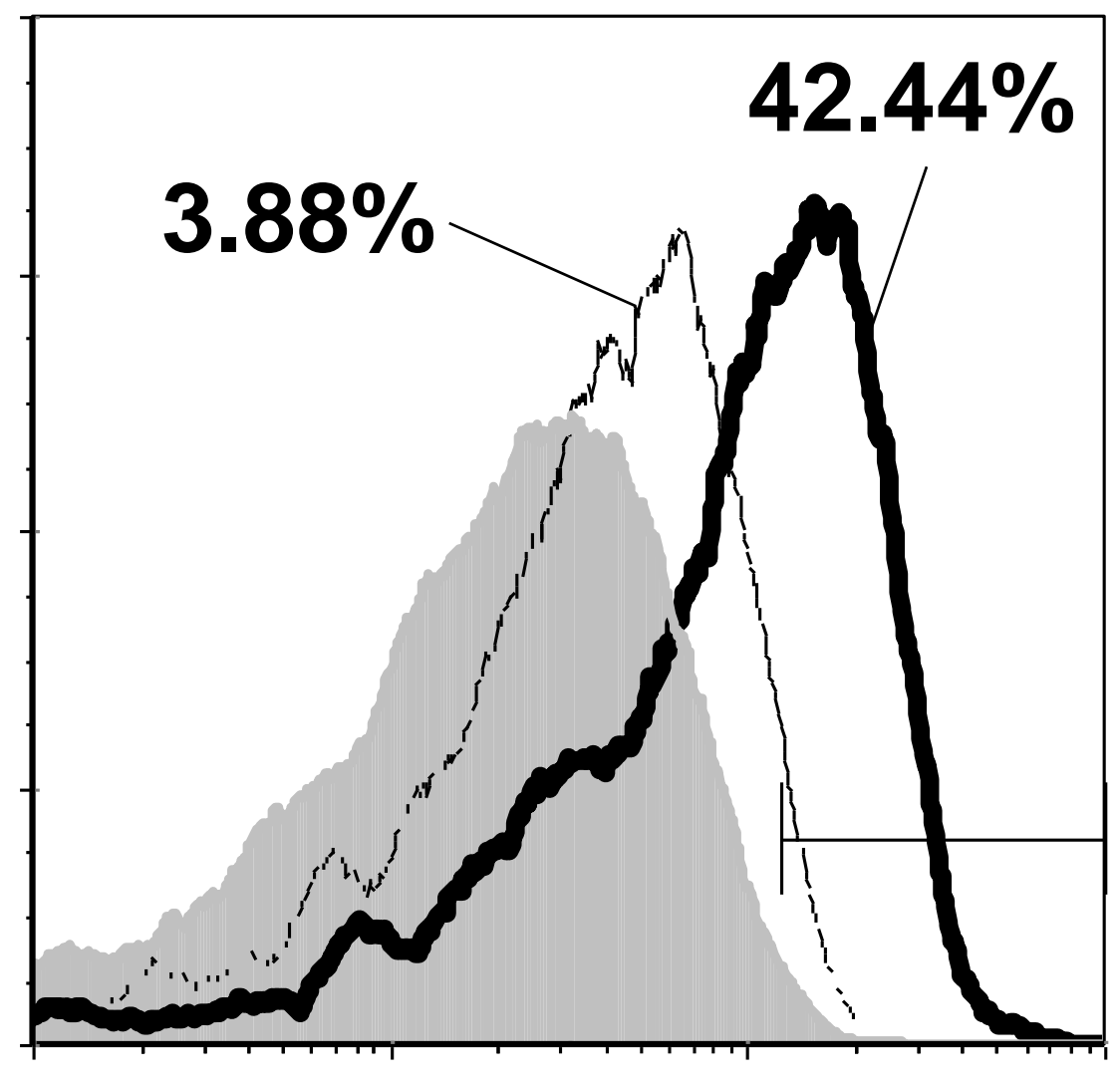

TUNEL

D

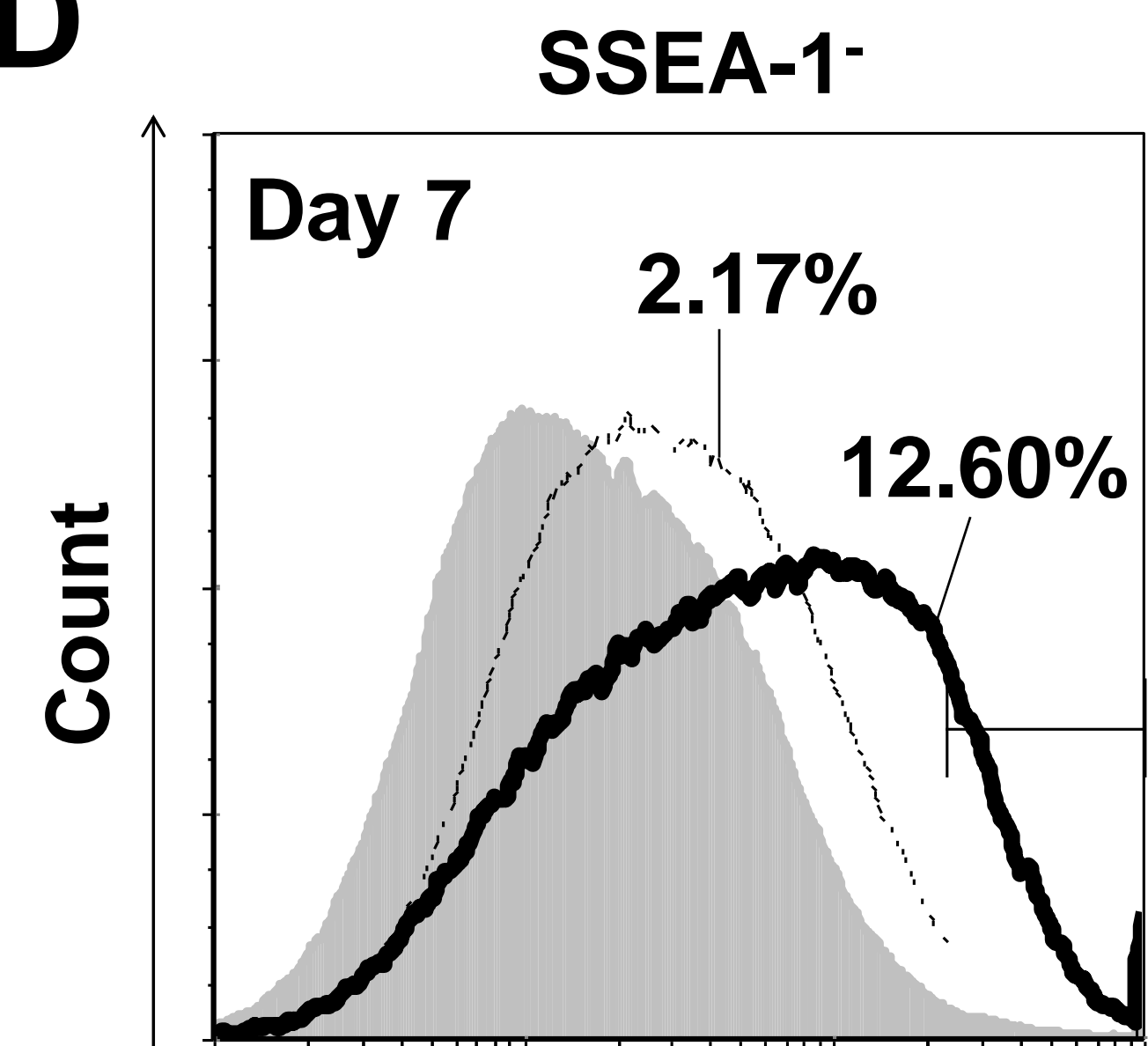

SSEA-1+

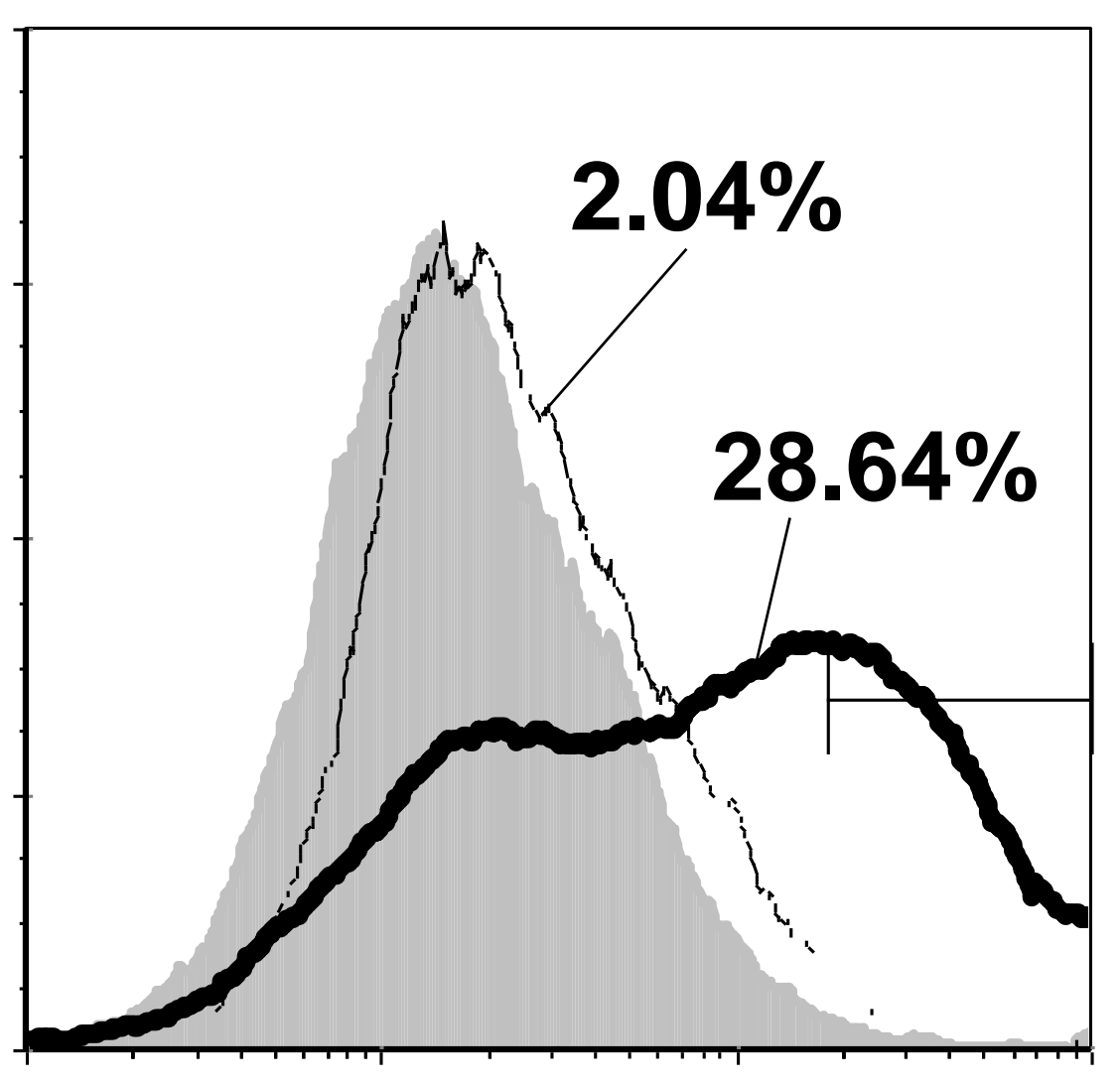

$$
\begin{aligned}
& \text { Untreated } \\
& - \text { TMZ }\left(200 \mathrm{mg} / \mathrm{m}^{2}\right) \\
& -\operatorname{scL-TMZ~}\left(75 \mathrm{mg} / \mathrm{m}^{2}\right)
\end{aligned}
$$

Untreated

TMZ $\left(200 \mathrm{mg} / \mathrm{m}^{2}\right)$

- scL-TMZ $\left(75 \mathrm{mg} / \mathrm{m}^{2}\right)$ 
Table 1. Sensitivity of GBM and other non-GBM tumor cells by TMZ and scL-TMZ.

\begin{tabular}{cccc}
\hline Cancer Type & Cell Line & TMZ IC $_{50}$ & scL-TMZ IC $_{50}$ \\
\hline Glioblastoma & U251 & $>50 \mu \mathrm{M}$ & $15.3 \mu \mathrm{M}$ \\
Glioblastoma & U87 & $>50 \mu \mathrm{M}$ & $19.6 \mu \mathrm{M}$ \\
Glioblastoma (TMZ-R) & U87R & $1357 \mu \mathrm{M}$ & $33.6 \mu \mathrm{M}$ \\
Glioblastoma (TMZ-R) & T98G & $>1500 \mu \mathrm{M}$ & $42.4 \mu \mathrm{M}$ \\
Lung & A549 & $>100 \mu \mathrm{M}$ & $38.6 \mu \mathrm{M}$ \\
Prostate & DU145 & $>100 \mu \mathrm{M}$ & $70.1 \mu \mathrm{M}$ \\
Pancreas & PANC-1 & $>100 \mu \mathrm{M}$ & $52.3 \mu \mathrm{M}$ \\
Breast & MDA-MB-231 & $>100 \mu \mathrm{M}$ & $30.1 \mu \mathrm{M}$ \\
Melanoma & MDA-MB-435 & $>100 \mu \mathrm{M}$ & $45.8 \mu \mathrm{M}$ \\
Colorectal & HT-29 & $>100 \mu \mathrm{M}$ & $39.1 \mu \mathrm{M}$ \\
\hline
\end{tabular}

TMZ-R: TMZ resistant. 
Table 2. Effect of TMZ and scL-TMZ on the hematological and biochemical parameters in mice on Day 7, 3 days after the 5 consecutive daily treatments with either 15 or $75 \mathrm{mg} / \mathrm{m}^{2} \mathrm{TMZ}$ dose.

\begin{tabular}{|c|c|c|c|c|c|}
\hline & \multirow{2}{*}{ Untreated } & TMZ & scL-TMZ & TMZ & scL-TMZ \\
\hline & & \multicolumn{2}{|c|}{$15 \mathrm{mg} / \mathrm{m}^{2}$ dose } & \multicolumn{2}{|c|}{$75 \mathrm{mg} / \mathrm{m}^{2}$ dose } \\
\hline \multicolumn{6}{|l|}{ Hematology } \\
\hline Leukocytes $\left(\times 10^{9} / \mathrm{L}\right)$ & $7.17 \pm 0.99$ & $5.88 \pm 0.58$ & $6.3 \pm 0.51$ & $4.72 \pm 0.99(L)$ & $8.25 \pm 0.35$ \\
\hline Neutrophils $\left(\times 10^{9} / \mathrm{L}\right)$ & $0.91 \pm 0.12$ & $0.99 \pm 0.17$ & $0.88 \pm 0.08$ & $0.79 \pm 0.15$ & $2.46 \pm 0.38(\mathrm{H})$ \\
\hline Lymphocytes $\left(\times 10^{9} / \mathrm{L}\right)$ & $5.97 \pm 0.88$ & $4.48 \pm 0.45$ & $4.89 \pm 0.37$ & $3.35 \pm 0.82(L)$ & $5.62 \pm 0.19$ \\
\hline Thrombocytes $\left(\times 10^{9} / \mathrm{L}\right)$ & $941.33 \pm 20.18$ & $970.00 \pm 105.00$ & $1059.67 \pm 95.75$ & $627.80 \pm 46.76(\mathrm{~L})$ & $949.75 \pm 80.55$ \\
\hline \multicolumn{6}{|l|}{ Blood Chemistry } \\
\hline $\operatorname{ALP}(\mathrm{U} / \mathrm{L})$ & $104.00 \pm 20.66$ & $99.60 \pm 9.46$ & $91.80 \pm 13.46$ & $72.60 \pm 10.54(\mathrm{~L})$ & $83.25 \pm 3.90$ \\
\hline ALT (U/L) & $37.00 \pm 4.36$ & $34.20 \pm 3.62$ & $37.40 \pm 1.86$ & $59.40 \pm 17.30(\mathrm{H})$ & $32.75 \pm 3.25$ \\
\hline AST (U/L) & $199.50 \pm 46.50$ & $184.20 \pm 46.07$ & $175.20 \pm 25.85$ & $333.20 \pm 83.20(\mathrm{H})$ & $150.00 \pm 24.09$ \\
\hline $\mathrm{BUN}(\mathrm{mg} / \mathrm{dL})$ & $17.00 \pm 2.00$ & $19.20 \pm 0.73$ & $17.60 \pm 1.03$ & $21.40 \pm 1.17(H)$ & $20.75 \pm 2.14$ \\
\hline Creatinine (mg/dL) & $0.17 \pm 0.07$ & $0.20 \pm 0.10$ & $0.14 \pm 0.02$ & $0.14 \pm 0.04$ & $0.18 \pm 0.03$ \\
\hline Total protein $(\mathrm{g} / \mathrm{dL})$ & $5.70 \pm 0.30$ & $5.94 \pm 0.17$ & $6.34 \pm 0.33$ & $6.02 \pm 0.17$ & $6.03 \pm 0.32$ \\
\hline Sodium $(\mathrm{mEq} / \mathrm{L})$ & $149.33 \pm 8.17$ & $155.00 \pm 2.12$ & $163.00 \pm 6.00$ & $161.40 \pm 3.41$ & $170.00 \pm 12.13$ \\
\hline Potassium (mEq/L) & $8.97 \pm 1.24$ & $8.20 \pm 0.34$ & $8.90 \pm 0.45$ & $10.28 \pm 0.89$ & $8.30 \pm 0.43$ \\
\hline CPK (U/L) & $472.50 \pm 133.50$ & $393.6 \pm 159.42$ & $546.00 \pm 270.23$ & $2973.80 \pm 1365.47(\mathrm{H})$ & $230.00 \pm 46.15(\mathrm{~L})$ \\
\hline
\end{tabular}

Values are mean \pm SD for 5 mice in each group. ALP: alkaline phosphatase, ALT: alanine transaminase, AST: aspartate aminotransferase, BUN: blood urea nitrogen, CPK: creatine phosphokinase. Values in bold letter represent more than $25 \%$ change from the untreated base line. (L): lower,

(H): higher. 